\title{
A Profile-based Two-level Pointer forwarding Cache Scheme for Reducing Location Management Cost in Wireless Mobile Networks
}

\author{
Md. Mohsin Ali, Md. Ziaur Rahman Khan, and Md. Ashraful Alam \\ Department of Computer Science and Engineering (CSE) \\ Khulna University of Engineering \& Technology (KUET) \\ Khulna - 9203, Bangladesh
}

\begin{abstract}
In this paper we propose an efficient location management strategy called the profile-based two-level pointer forwarding cache scheme to improve the overall performance of the wireless mobile networks. It uses the two-level pointer for location update and the profile-based cache for call delivery. Location update for each Mobile Terminal (MT) is managed by the nearby Visitor Location Register (VLR) until it crosses the threshold of the number movements between Registration Areas (RAs). This reduces the frequency of accessing the remote Home Location Register (HLR) which eventually minimizes the location update cost by distributing its work. On the other hand, a profile of the MT's calling pattern is maintained in HLR and it is copied in the VLR caches from where majority of the calls are generated. These caches are searched first to find out the called MT's location in order to deliver a call. This delivery cost will be reduced if the location information is found in the caches since it does not have to access the remote HLR for searching the location information. The analytical modeling and numerical results show that the proposed method outperforms all other previous methods in terms of network signaling traffic load irrespective of the MTs' mobility and calling pattern.
\end{abstract}

\section{Categories and Subject Descriptors}

D.3.3 [Programming Languages]: Language Contructs and Features - abstract data types, polymorphism, control structures.

\section{General Terms}

Mobile Computing, Wireless Communications.

\section{Keywords}

Pointer forwarding, profile-based cache, location update cost, call delivery cost, HLR, VLR.

\section{INTRODUCTION}

Location management is the most important issues in the current as well as next-generation wireless mobile networks. It is concerned with the issues of tracking and finding MTs in order to roaming in the network coverage area. This tracking in all wireless communication systems is currently managed by two types of standards such as basic IS-41 [1] and GSM [2]. Both of these architectures are based on a two-level database hierarchy called HLR and VLR which are used to store the MTs' location information. The whole network coverage area under these architectures is divided into same sized and shaped cells. Each cell has a Base Transceiver Station (BTS) through which MTs of the cell communicate through a wireless link. The cells are grouped together to form larger areas called RAs. All the BTSs belonging to a given RA are wire-connected to a Mobile
Switching Center (MSC) and the MTs are wireless-connected to these BTS. Sometimes the VLR may co-locate with the MSC and a group of RAs are interfaced with the Local Signaling Transfer Point (LSTP) following the HLR. An HLR is the centralized database that contains the records of all MTs' profiles together with the location information for the entire network. Similarly, each VLR stores replications of the user profiles of the subscribers currently residing in its corresponding RA. However, there may exist one or more HLRs in the network depending on its configuration. Figure 1 shows the architecture of the wireless mobile networks.

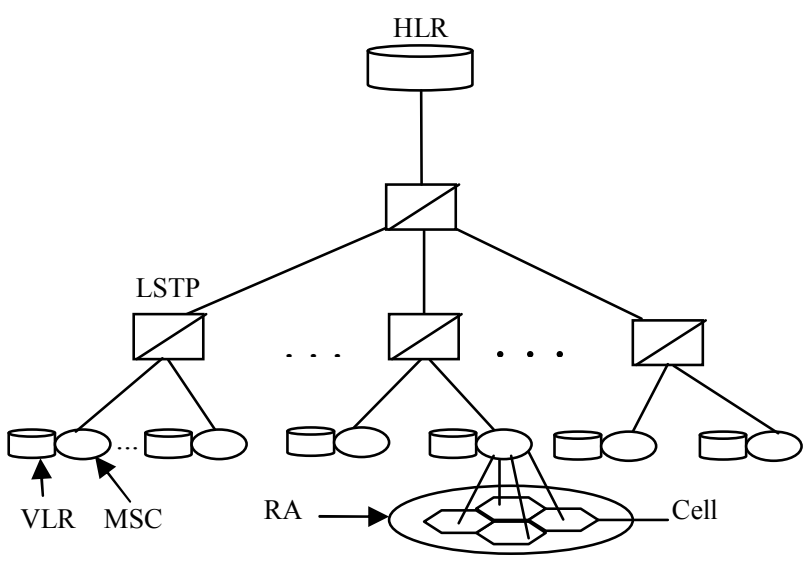

Figure 1. Wireless mobile networks architecture.

Two basic operations in location management are location registration or update and call delivery. The former one is the process of informing the network about the MT's current location information; whereas the latter one is the process of determining the serving VLR and the cell location of the called MT just before the connection setup between the caller and called MTs. An MT needs to inform its new location information to the MSC whenever it enters into a new RA. The MSC updates its corresponding VLR and transmits the message combined with this new information to the HLR. The HLR confirms the MSC about this successful registration and also sends a location deregistration signaling message to the MT's old VLR in the corresponding RA prior to receive an acknowledgement. In order to deliver a call to the called MT, the HLR is queried to determine the serving MSC of the called MT. After this successful query, the HLR sends a signaling message to this MSC in order to determine the serving BTS of the called MT by exploring all cells within the corresponding RA [3]. 
As the number of MTs within the network and their service requirements are exploding rapidly day-by-day, location management under the basic IS-41 can not sufficiently support these MTs. For instance, increased users and their requirements make the network overwhelm by exchanging enormous network signaling message as well as make the location server congested. In order to overcome these problems, several approaches are proposed in terms of designing signal exchanging algorithms and network architectures. As a part of this, local anchoring scheme [4], per-user pointer forwarding scheme [5], [6], two-level pointer forwarding scheme (TWO LEVEL) [7], pointer forwarding based local anchoring (POFLA) scheme [8] and profile-based location caching scheme [9], [10] are already proposed to minimize the network signaling overhead in the network in order to serve the user.

A new location management strategy is proposed in this paper by effectively utilizing the MTs' mobility and calling pattern. The mobility pattern is effectively managed by the pointer forwarding based local anchoring (POFLA) scheme [8] where some VLRs are selected as Mobility Agent (MA) for managing local location registration locally and two types of pointers are used for pointing MTs' location from these MAs. A pointer is setup from the old VLR to the new one without updating the MA when it crosses the RA boundary and enters in another RA under the control of the separate VLR. The current serving VLR is selected as the new MA instead of the previous one if the first type of the pointer reaches its threshold and the MT updates its location information directly to the HLR when the second type of the pointer reaches its threshold. In this way it manages the local location registration locally instead of informing the HLR. As a result the location registration cost is minimized. On the other hand, the MTs' calling pattern is effectively managed by the HLR and it is cached to the VLR from where majority of the calls are originated [10]. These caches are searched first to deliver a call to the called MT and it reduces the call delivery cost significantly in case of cache hit. In order to achieve the benefit of these two approaches, we propose a method by combining these two methods with some modification which shows better performance irrespective of the MTs' calling and mobility pattern.

The rest of the paper is organized as follows. Section 2 provides an overview of the existing related research work. Our proposed approach is described in section 3. Section 4 provides the analytical modeling of the related and proposed approaches. Numerical results and comparisons among different approaches based on some experimental results are described in section 5 . We provide a concluding remark in section 6 .

\section{RELATED WORK}

There are considerable amount of work have been done on the location management to improve the overall performance of the wireless mobile networks [4], [5], [6], [7], [8], [9], [10].

A local anchoring scheme is proposed in [4] where a VLR close to a mobile user is selected as local anchor for its MT and performs its location update to this local anchor when it moves from one RA to another. The VLR retains as local anchor for this MT until it receives a call request from another MT. The new serving VLR will take the responsibility as new local anchor as soon as it receives a new call served from this VLR and a location update will be sent to the HLR. This scheme avoids the frequent HLR update with the help of increased local signaling traffic. The local anchor update may become costly whenever the user constantly moving from one RA to another without receiving any call request. This scheme is converted to dynamic local anchoring scheme if the local anchors are selected dynamically from the user expected future locations. However, the call delivery procedure of this strategy is the same as that of the IS-41 strategy.

A per-user pointer forwarding scheme is proposed in [5] where a pointer is setup from the previous VLR to the current one when a MT moves from one RA to another one instead of informing to the HLR. The MT's locations are first searched in the HLR and then traced through this pointer chain during call delivery to the mobile users. This strategy is suitable for the system with high user mobility rate. Although the location registration or update cost in this scheme is smaller but needs more time to deliver a call through the longer pointer chain.

A forwarding pointer-based cache scheme is proposed in [6] where one-level pointer is used to manage the local location registration locally by saving accessing the congested HLR which eventually reduces location registration or update cost. It also applies the location caching in the VLR so that the called MTs' location can be traced from this cached information without going to the HLR. It does not need to directly query the HLR in case of cache miss like conventional per-user location caching as it can find that location information through pointer chain available in the VLR. As a result, it can reduce the call delivery cost more compared to the basic per-user location caching strategy.

A two-level pointer forwarding scheme (TWO LEVEL) is proposed in [7] which uses two types of pointer together with some VLRs serving as MAs for managing local location registration locally. A level 2 pointer is setup from the old VLR to the new one if the MT crosses the RA boundary and updates its location to the current MA instead of HLR. The current VLR is selected as MA if its level 2 pointer chain reached its threshold and a level_1 pointer is setup from the previous MA to the current one. As a result, the location registration or update cost is minimized as it needs smaller access to the HLR. In order to deliver a call, it first searches in the HLR and then follows the level_2 and level_1 pointers to find out the called MTs' location.

A pointer forwarding based local anchoring (POFLA) scheme is proposed in [8] which is almost similar to that of the two-level pointer forwarding scheme. The only major difference is that there is only one MA exist in the whole network and leve 2 and level_1 pointers are renamed with L-pointer (low pointer) and Hpointer (high pointer) respectively. An H-pointer is setup from the current MA to the new VLR if L-pointer reaches its threshold during RA crossing and does not perform location update in HLR. The current serving VLR is selected as a new MA replacing the previous $\mathrm{MA}$ if $\mathrm{H}$-pointer reaches its threshold during $\mathrm{RA}$ boundary crossing and inform the MA's new location to the HLR. On the other hand, the call delivery procedure is started from searching in the HLR following H-pointer and L-pointer. Although this method reduces the location registration time, but call delivery needs more time for searching through the longer pointer chain.

A profile based scheme is proposed in [9] where a user's daily routine information is stored in the profile. If the user properly follows his or her routine then there is no need to send location update message in the network which reduces the update network signaling traffic. The call delivery procedure involves searching 
all the VLR following the descending probability order. As a result, although the location update cost is reduced in this approach, but call delivery cost is increased significantly.

A profile-based location caching with fixed local anchor scheme is proposed in [10] where a VLR is selected as fixed local anchor for a certain region to manage the local location registration locally instead of informing to HLR. This eventually minimizes the location registration or location update cost. On the other hand, MTs' calling pattern maintained by the HLR is cached to the VLRs from where majority of the calls are generated. These VLR caches are searched first for finding the called MTs' location information instead of directly going to the HLR. This significantly reduces the call delivery cost if cache hit occurs during forwarding the call to the called MTs.

\section{PROPOSED METHOD}

We propose a method called the profile-based two-level pointer forwarding cache scheme by combining the two approaches [8] and [10] with some modifications to improve the overall performance of the wireless mobile networks. In this approach, a VLR close to the MTs is selected as MA for these MTs to perform local location registration or update for these MTs to this MA. A VLR can also be selected as MA for an MT if this serving VLR forwards the incoming call to this MT attached to it as it knows the correct location information of that MT. When an MT crosses RA boundaries, it informs its location information to the new VLR and a pointer called L-pointer is setup from the old VLR to the new one without informing to the MA and HLR. Upon reaching the L-pointer to its threshold value, the new VLR updates the MT's new location information to the current MA. This threshold value initiates setting up the H-pointer from the MA to the new VLR and resets the L-pointer value. The H-pointer may also be reached its threshold value. In that case, the MA for that MT is changed to the current VLR and resets both the Lpointer and H-pointer values. The current MA sends location update signaling message to the HLR and a profile of MTs calling pattern from this HLR is saved to the frequently call originated VLR caches. To deliver a call, the calling VLR cache is searched first for called MT's location information. This call is directly forwarded to the called MT without searching the HLR for the availability of the called MT's location information in the cache. The cache information is always up-to-date in this approach and there is no chance of becoming the cache information obsolete if the information is available in the cache. However, if this location information is absent in the VLR cache, this call is forwarded to the called MT after querying the called MT's location information directly into the HLR. Additionally, during forwarding the call to the MT attached to its serving VLR, the events that occur are the same as that of the events when H-pointer is reached its threshold value. The MA selections, location registration, call delivery, and call arrival process of the proposed method is shown in Figure 2.

\section{ANALYTICAL MODELING}

In order to compare the signaling costs of the proposed method with that of the existing methods, we only need to compare the signaling traffic in the interservice time. This time is defined as the interval between the end of the current call and the beginning of the next call [11], [12]. The notations used for the analysis is given in Table 1 and the analytical modeling in most of the cases resembles to the modeling discussed in [7] and [8].

\section{Procedure MA_Selectioin}

- A VLR close to the MTs are selected as MA for these MTs for managing local location registration.

- Reset L-pointer and H-pointer.

\section{Procedure Location_Registration}

IF (MT crosses the RA boundary and enters into the new VLR from the old one)

- An L-pointer is setup from the old VLR to the new one.

IF (L-pointer is reached its threshold)

- The new VLR updates user's location to the current MA.

- An H-pointer is setup from the MA to the current new VLR.

- Reset L-pointer.

IF (H-pointer is reached its threshold)

- Set the current new VLR as the new MA replacing the previous MA.

- Reset both L-pointer and H-pointer.

- New MA performs location update to the HLR.

- A profile of MTs' calling pattern maintained by the HLR is cached to the VLR from where majority of the calls are originated.

\section{Procedure Call_Delivery}

IF (A VLR receives an incoming call from the MT attached to it in order to deliver that call to the called MT)

- The VLR cache is searched to find out the called MT's location information.

IF (Location information of the called MT is found in the VLR cache)

- A connection is established between the caller and called MTs to deliver the call.

\section{ELSE}

- The HLR is searched to find out the called MT's location information and a connection is established between the caller and called MTs to deliver the call.

\section{Procedure Call Arrival}

IF (A VLR receives an incoming call to deliver it to the MT attached to it from another MT)

- Deliver this call to the called MT attached to that VLR.

- The serving VLR is selected as the new MA for the called MT replacing the previous one.

- Reset both L-pointer and H-pointer.

- New MA performs location update to the HLR.

- A profile of MTs' calling pattern maintained by the HLR is cached to the VLR from where majority of the calls are originated.

Figure 2. The location management procedure of the proposed approach.

The total location management costs during the interservice time for the two location management schemes are as follows.

$$
\begin{aligned}
& C=M+F=\frac{m}{\rho}+F \\
& C^{\prime}=M^{\prime}+F^{\prime}
\end{aligned}
$$


Table 1. The notations used in analytical modeling.

\begin{tabular}{|c|c|}
\hline Notation & Description \\
\hline$C$ & The total cost for the basic IS-41 scheme \\
\hline$C^{\prime}$ & The total cost for the proposed method \\
\hline$K_{1}$ & The threshold for the L-pointer chain \\
\hline$K_{2}$ & The threshold for the H-pointer chain \\
\hline$m$ & The average cost of location update to the HLR \\
\hline$M$ & $\begin{array}{l}\text { The total location update cost during the } \\
\text { interservice time in the IS- } 41 \text { scheme }\end{array}$ \\
\hline$F$ & The total cost of call delivery in the IS-41 scheme \\
\hline$M^{\prime}$ & $\begin{array}{l}\text { The total location updates cost in the proposed } \\
\text { scheme during the interservice time }\end{array}$ \\
\hline$F^{\prime}$ & The total call delivery cost in the proposed scheme \\
\hline$S_{1}$ & The pointer setup cost of an L-pointer \\
\hline$S_{2, \mathrm{j}}$ & The pointer setup cost of the $j$ th H-pointer \\
\hline$T_{1}$ & $\begin{array}{l}\text { The cost of traversing an L-pointer between two } \\
\text { adjacent VLRs }\end{array}$ \\
\hline$T_{2, \mathrm{j}}$ & The cost of traversing the $j$ th $\mathrm{H}$-pointer \\
\hline$\alpha(i)$ & $\begin{array}{l}\text { The probability that there are } i \text { RA crossings } \\
\text { during the interservice time }\end{array}$ \\
\hline$P$ & $\begin{array}{l}\text { The processing cost of setting up a pointer }(\mathrm{H} \text { or } \\
\mathrm{L})\end{array}$ \\
\hline$G$ & The signaling cost of setting up an L-pointer \\
\hline$\beta$ & $\begin{array}{l}\text { The coefficient of signaling cost for an H-pointer } \\
(\beta \geq 1)\end{array}$ \\
\hline$\rho$ & The user call-to-mobility ratio (CMR) \\
\hline$N$ & $\begin{array}{l}\text { The number of VLRs in which HLR sends user } \\
\text { profile }\end{array}$ \\
\hline$P_{h}$ & The probability of cache hit \\
\hline$P_{c}$ & $\begin{array}{l}\text { The signaling cost for profile caching in the } \\
\text { VLT(s) from the HLR }\end{array}$ \\
\hline$g$ & $\begin{array}{l}\text { The residence time of the mobile user at a } \\
\text { registration area }\end{array}$ \\
\hline
\end{tabular}

The pointer setup and traversing costs of the L-pointers for the proposed method are as follows.

$$
\begin{aligned}
& S_{1}=G+P \\
& T_{1}=\frac{1}{2} G+P
\end{aligned}
$$

The costs of the H-pointers are not fixed values as the length of the H-pointers changes with the user's location. So the pointer setup and traversing costs of the H-pointers are as follows.

$$
\begin{aligned}
& S_{2, j}= \begin{cases}0, & \text { if } j=0 \\
j \beta G+P, & \text { Otherwise }\end{cases} \\
& T_{2, j}= \begin{cases}0, & \text { if } j=0 \\
\frac{1}{2} j \beta G+P, & \text { Otherwise }\end{cases}
\end{aligned}
$$

Where, $j$ means pointer setup cost or traversing cost for the $j$ th $\mathrm{H}-$ pointer.

Now the formula for $M^{\prime}$ and $F^{\prime}$ can be derived as follows. Suppose that a user crosses $i$ RA boundaries during the interservice time. The HLR is updated $\left\lfloor i / K_{1} K_{2}\right\rfloor$ times, the Lpointer setup is occurred $i-\left\lfloor i / K_{1}\right\rfloor$ times, H-pointer setup cost is
$\sum_{j=0}^{K_{2}-1} S_{2, j}$ for each HLR update. In addition, there are $\left\lfloor i-\left\lfloor i / K_{1} K_{2}\right\rfloor K_{1} K_{2} / K_{1}\right\rfloor$ remaining times H-pointer setup is occurred and $\left\lfloor i / K_{1} K_{2}\right\rfloor$ times VLR cache is updated with MTs calling pattern profile from the HLR. Moreover, to deliver a call, there are $\left\lfloor i-\left\lfloor i / K_{1} K_{2}\right\rfloor K_{1} K_{2} / K_{1}\right\rfloor$ H-pointer and ( $\left.i-\left\lfloor i / K_{1}\right\rfloor K_{1}\right)$ L-pointer needs to traverse. So the equations for $M$ ' and $F^{\prime}$ are as follows.

$$
\begin{aligned}
M^{\prime}= & \sum_{i=0}^{\infty}\left\{\left\lfloor i / K_{1} K_{2}\right\rfloor m+\left(i-\left\lfloor i / K_{1}\right\rfloor\right) S_{1}+\left\lfloor i / K_{1} K_{2}\right\rfloor\right. \\
& \left.\times\left(\sum_{j=0}^{K_{2}-1} S_{2, j}\right)+\sum_{j=0}^{\left\lfloor i-\left\lfloor i / K_{1} K_{2}\right\rfloor K_{1} K_{2} / K_{1}\right\rfloor} S_{2, j}+\left\lfloor i / K_{1} K_{2}\right\rfloor P_{c}\right\} \alpha(i) \\
F^{\prime}= & P_{h}(F / 2)+\left(1-P_{h}\right)\left\lfloor F+\sum_{i=0}^{\infty}\left\{T_{2,\left\lfloor i-\left\lfloor i / K_{1} K_{2}\right\rfloor K_{1} K_{2} / K_{1}\right\rfloor}\right.\right. \\
& \left.\left.+\left(i-\left\lfloor i / K_{1}\right\rfloor K_{1}\right) T_{1}\right\} \alpha(i)\right]
\end{aligned}
$$

Here $(F / 2)$ is the call delivery cost of the proposed method when location information is found in the cache since it is assumed that this cost will be half compared to that of the IS-41 scheme.

The signaling cost for profile caching in the VLT(s) from the $\mathrm{HLR}, P_{c}$ is defined as follows.

$$
P_{c}=N \times\left(\frac{m}{2}\right)
$$

Since this signaling is transmitted only in one direction, from HLR to VLR(s), its cost will be the half $(\mathrm{m} / 2)$ compared to the location update cost to the HLR (m) that also needs confirmation.

In order to evaluate $\alpha(i)$, the followings are assumed.

1. The interservice time is exponentially distributed with average $1 / \lambda$.

2. The residence time of the mobile user at an RA is a random variable with general probability density function $f_{m}(t)$ and the Laplace transform with average RA residence time $1 / \mu$.

So for simplicity, we denote $g=f_{m}^{*}(\lambda)$. We assume that the RA residence time follows Gamma distribution with mean $1 / \mu$. From the Laplace transform of this Gamma distribution, $g$ is defined as follows.

$$
g=\left(\frac{\gamma \mu}{\lambda+\gamma \mu}\right)^{\gamma}=\left(\frac{\gamma}{\rho+\gamma}\right)^{\gamma}
$$

In particular, when $\gamma=1$, we have an exponential distribution of the RA residence time and in this case $g$ is defined as follows.

$$
g=\frac{1}{\rho+1}
$$


Based on the above assumptions, the probability $\alpha(i)$ is defined as follows (see [13] for detailed derivation).

$$
\alpha(i)=\frac{(1-g)^{2} g^{i-1}}{\rho}
$$

Finally we obtain the following equations for $M^{\prime}$ and $F^{\prime}$ (see [8] for detailed derivation).

$$
\begin{aligned}
M^{\prime}= & \frac{G+P}{\rho}-\frac{(1-g)(G+P) g^{K_{1}-1}}{\rho\left(1-g^{K_{1}}\right)} \\
& +\frac{\left(K_{2}-1\right) K_{2} \beta G+2\left(K_{2}-1\right) P+2 m}{2} \times \frac{(1-g) g^{K_{1} K_{2}-1}}{\rho\left(1-g^{K_{1} K_{2}}\right)} \\
& +\frac{\beta G(1-g)}{2 \rho g\left(1-g^{K_{1} K_{2}}\right)\left(1-g^{K_{1}}\right)^{2}} \times\left\{g^{K_{1}}+g^{2 K_{1}}-K_{2}^{2} g^{K_{1} K_{2}}\right. \\
& \left.+\left(2 K_{2}{ }^{2}-2 K_{2}-1\right) g^{K_{1}\left(K_{2}+1\right)}-\left(K_{2}-1\right)^{2} g^{K_{1}\left(K_{2}+2\right)}\right\} \\
& +\frac{(\beta G+2 P)(1-g)\left[\left(K_{2}-1\right) g^{K_{1}\left(K_{2}+1\right)}-K_{2} g^{K_{1} K_{2}}+g^{K_{1}}\right]}{2 \rho g\left(1-g^{K_{1} K_{2}}\right)\left(1-g^{K_{1}}\right)} \\
& +N \times\left(\frac{m}{2}\right) \frac{(1-g) g^{K_{1} K_{2}-1}}{\rho\left(1-g^{K_{1} K_{2}}\right)} \\
F^{\prime}= & P_{h} \times\left(\frac{F}{2}\right)+\left(1-P_{h}\right)\left[F+\frac{G+2 P}{2 \rho}\left\{1-\frac{K_{1}(1-g) g^{K_{1}-1}}{\left(1-g^{K_{1}}\right)}\right\}\right. \\
& +\frac{P(1-g)\left(g^{K_{1}-1}-g^{K_{1} K_{2}-1}\right)}{\rho\left(1-g^{K_{1} K_{2}}\right)} \\
& +\frac{\beta G(1-g)\left\{\left(K_{2}-1\right) g^{K_{1}\left(K_{2}+1\right)}-K_{2} g^{K_{1} K_{2}}+g^{K_{1}}\right\}}{2 \rho g\left(1-g^{K_{1} K_{2}}\right)\left(1-g^{K_{1}}\right)}
\end{aligned}
$$

On the other hand, the set of equations for the pointer forwarding based local anchoring (POFLA) scheme [8], and the two-level pointer forwarding scheme (TWO LEVEL) [7] are derived and shown in [7] and [8]. The set of equations for the dynamic local anchoring scheme [4] is derived from [8] by setting $K_{1}=1$.

\section{NUMERICAL RESULTS AND COMPARISONS}

We assume that all the message sizes are equal for all signaling transactions as we only compare the relative performance of the aforementioned schemes with the proposed one. Since all the location update and call delivery for IS-41 and GSM procedure involve same number of messages exchange between the HLR and VLR both, we choose $m=F=1$. We also normalize $N=1$ and $P_{h}=0.5$ like existing papers. To setup the threshold for Lpointer and H-pointer of the proposed method, POFLA method, and TWO LEVEL method, we assume $K_{1}=K_{2}=3$. We also set $K_{2}=9$ for the DLA method to make the comparison among all the methods fair since $K_{l}=1$ for this approach and we need to traverse total 9 pointers for forwarding the call.

Figure 3 shows the relative location update, call delivery, and net costs of the proposed, POFLA, TWO LEVEL, and DLA methods with respect to MT's CMR for $P=0.05, G=0.1$, and $\beta=1.5$. It is observed from Figure 3(a) that the proposed method generates higher location update cost than the POFLA method. This is true

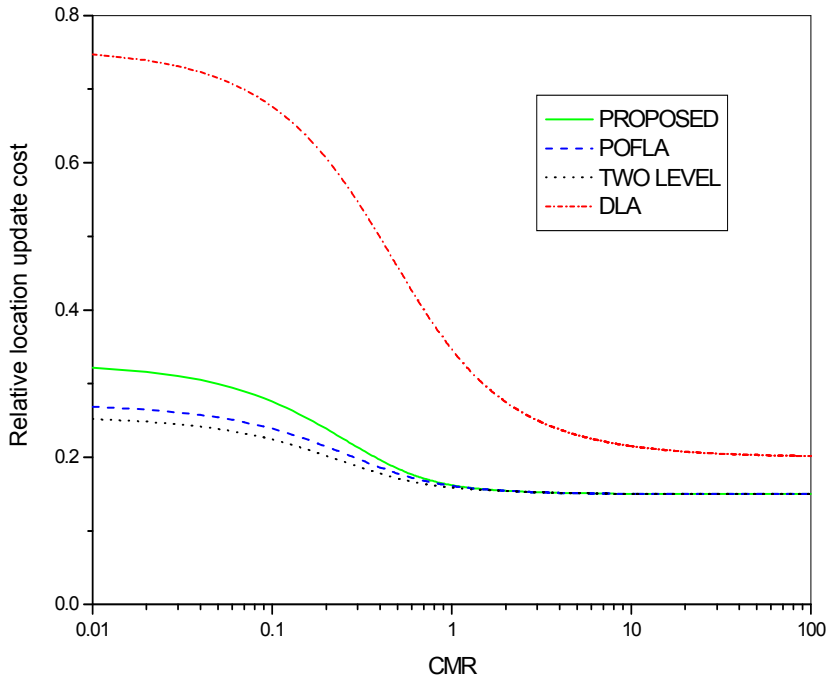

(a) Location update cost
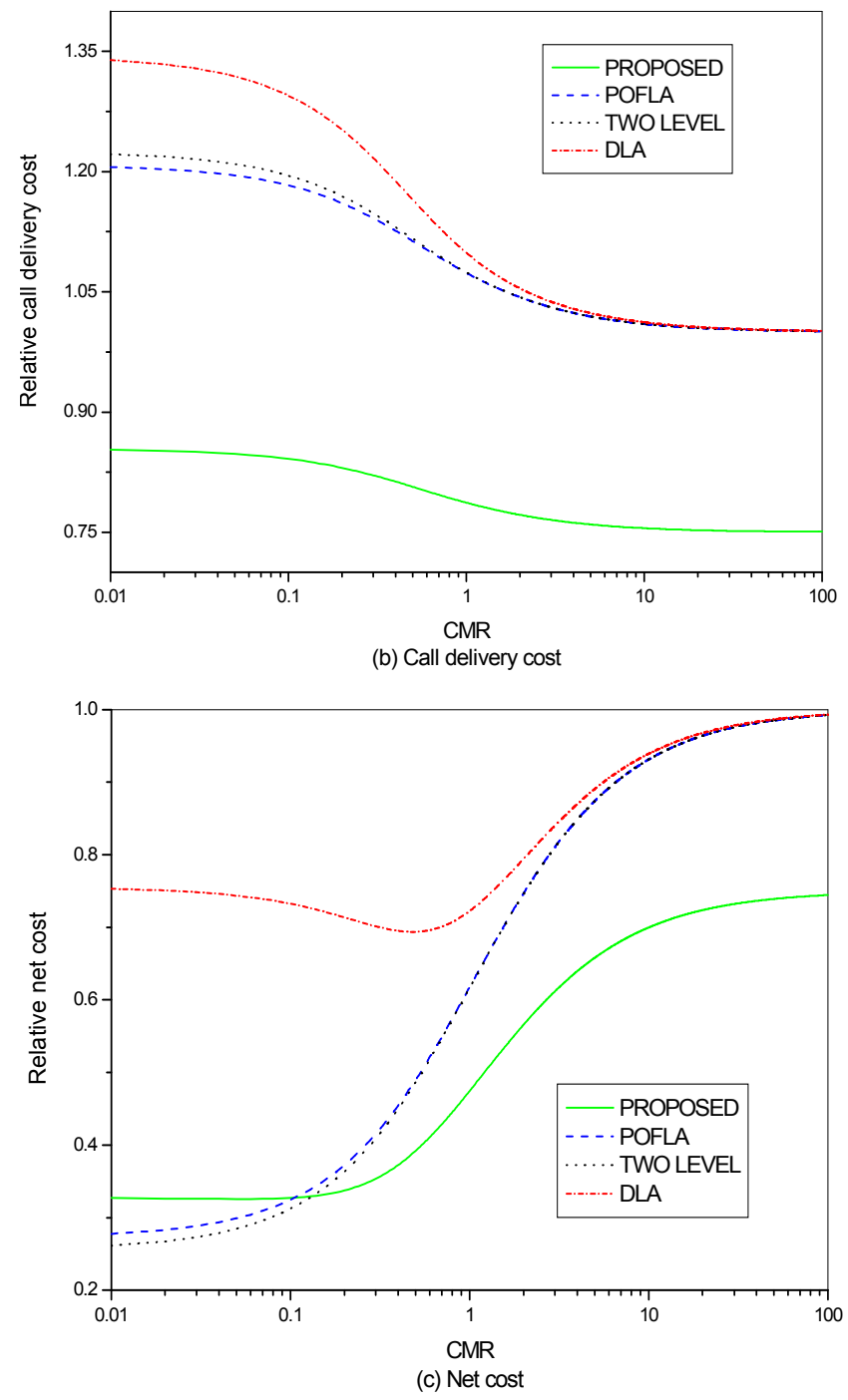

Figure 3. The relative costs of the four schemes with $P=0.05$, $G=0.10$ and $\beta=1.5$. 


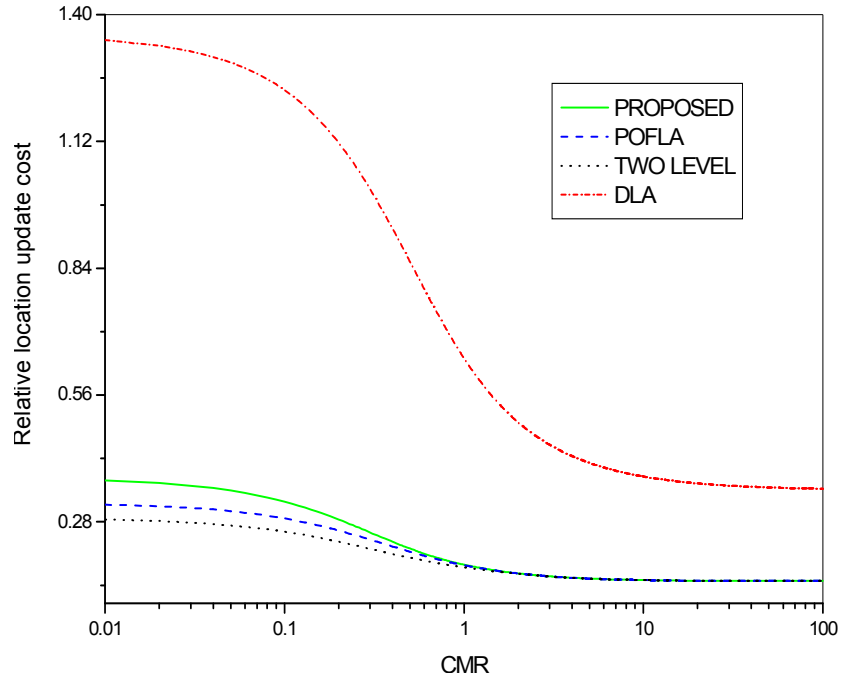

(a) Location update cost

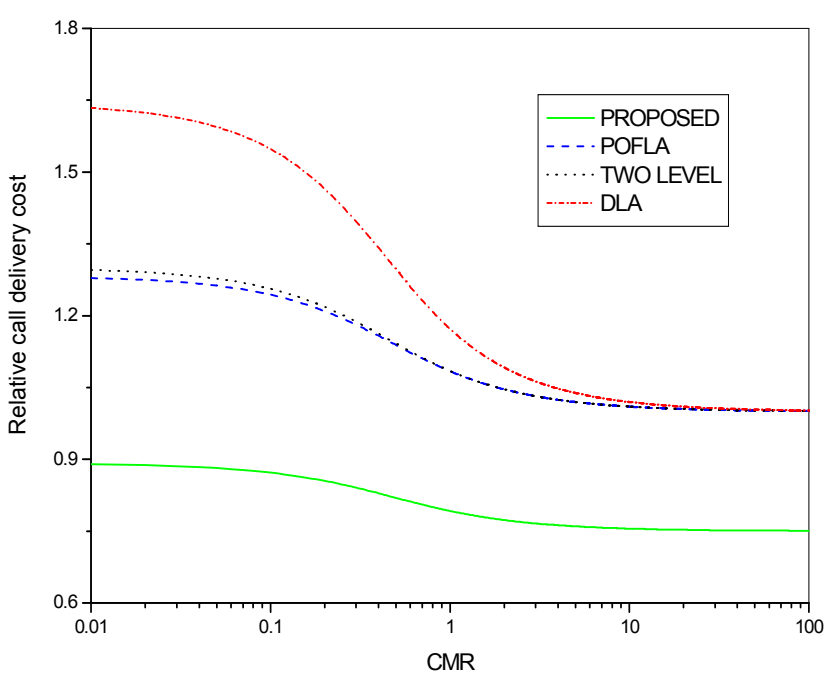

(b) Call delivery cost

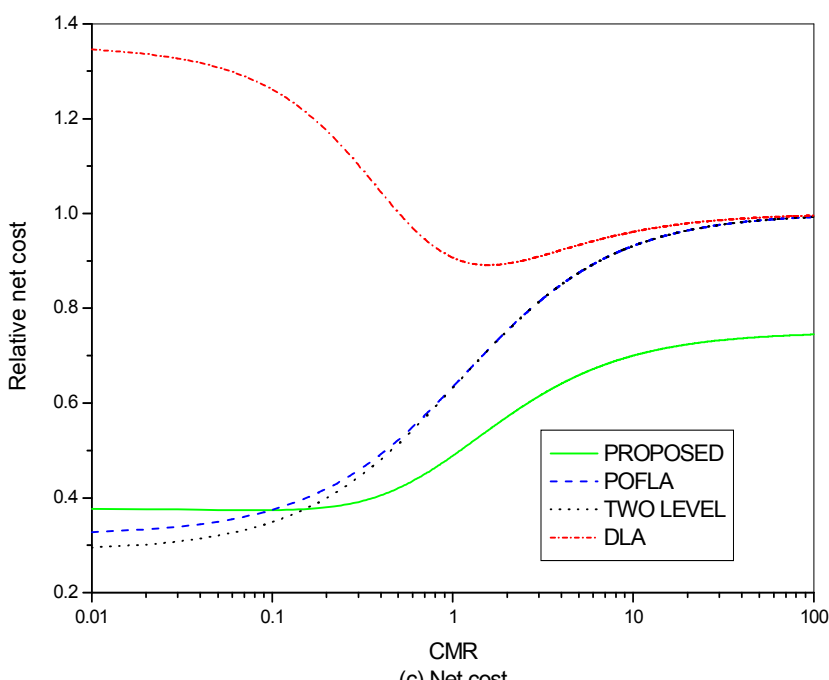

(c) Net cost

Figure 4. The relative costs of the four schemes with $P=0.05$, $G=0.10$ and $\beta=3.0$.

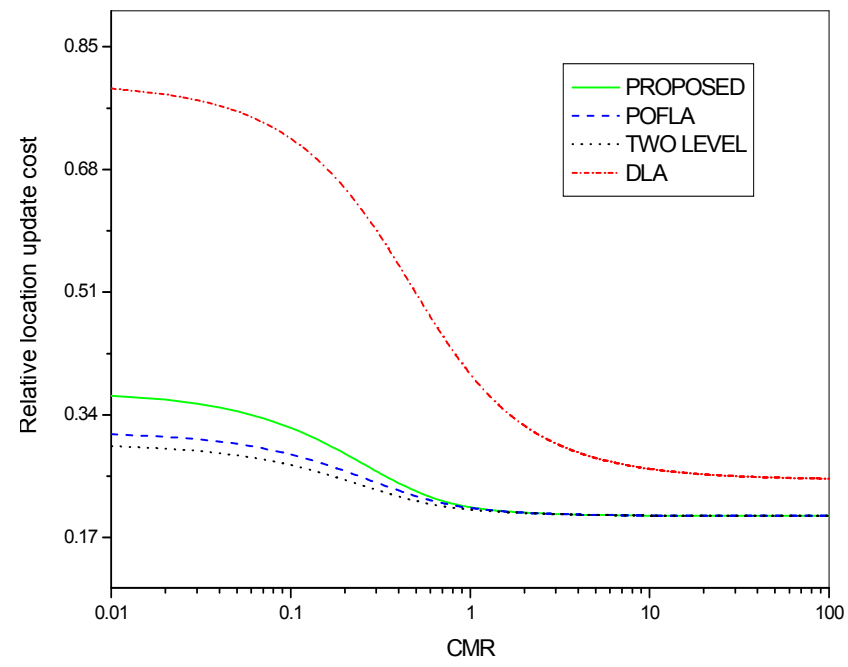

(a) Location update cost

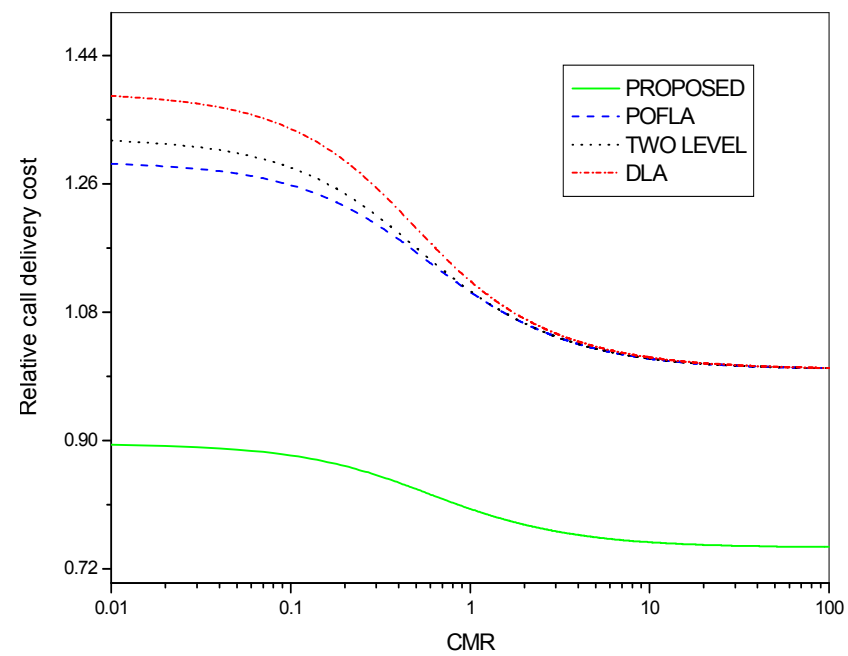

(b) Call delivery cost

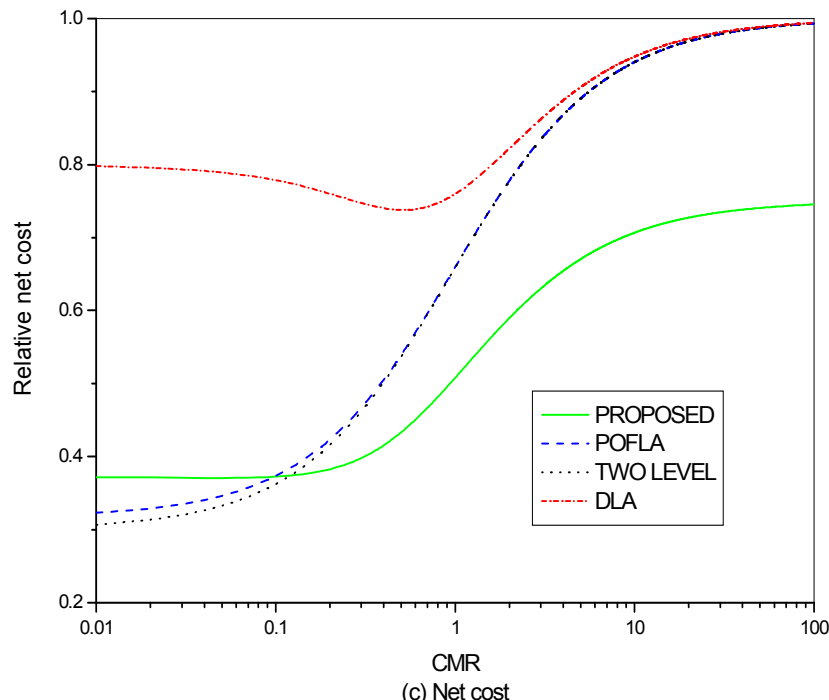

Figure 5. The relative costs of the four schemes with $P=0.10$, $G=0.10$ and $\beta=1.5$. 
and easily explainable as the proposed method generates some extra signaling cost in the network for caching the profile in the VLR(s) from the HLR when H-pointer reached its threshold value. For the TWO LEVEL scheme, the level_2 pointer is the Lpointer and level_1 pointer is the H-pointer and they are usually shorter than the L-pointer and H-pointer values. As a result, TWO LEVEL generates smaller location update costs than other methods. However, DLA shows highest location update cost as the decision of the local anchor changing is made based on the user's next event which is derived according to the user's mobility pattern. On the other hand, from the relative call delivery costs of the four methods shown in Figure 3(b) demonstrate that the proposed method outperforms all other methods irrespective of the CMR values. The proposed method has to traverse a few pointers for delivering the call if it finds the called MT's location information in the VLR cache which makes its performance better. Moreover, the relative net cost of the proposed and existing methods shown in Figure 3(c) make us clear that the proposed method outperforms all other methods for CMR value greater than 0.1 . The reason behind this result is that the location registration becomes dominated over call delivery for small values of CMR and the proposed methods generates more network signaling traffic for sending user profile from the HLR to the VLR caches with this increased location registration. Therefore, the net cost of the proposed method for the small CMR values increases. However, call delivery becomes dominated for higher values of CMR and the proposed method generates low net cost as it finds the called MT's location in the cache to deliver the call rather than searching this location in the HLR.

We increase the signaling transfer coefficient $\beta$ from 1.5 to 3.0 in Figure 4 which means that the H-pointer setup cost is higher. Under this condition, the location update cost, call delivery cost, and the net cost of all the schemes are increased. Although the $\mathrm{H}-$ pointer setup cost increases, the proposed method outperforms other related methods.

Figure 5 shows the performance of the four methods when the pointer processing cost $P$ is increased to 0.1 . With the increase of user population and the mobility, the processing cost for pointer management would increase too. The processing cost also includes database transaction costs and may generate extra delay with large number of operational delays. We can also observe that the proposed method outperforms all other related methods although the costs for all methods are increased under this condition.

\section{CONCLUSION}

Location management is one of the most important issues in current and next-generation wireless mobile networks consisting of two basic operations - location update and call delivery. With the increase of mobile users and their mobility, the network signaling traffic due to these two basic operations is increasing day-by -day. In order to solve this problem, this paper proposes an efficient location management strategy by simply combining two strategies - one of which significantly reduces location update cost and another one minimizes call delivery cost. As a result, the total network signaling traffic due to location update and call delivery reduces significantly compared to existing approaches. The analytical modeling and numerical results obtained for these approaches also verifies that the proposed method exploits all other previous methods.
We are currently working to improve this approach by combining other approaches like time-based approach to it.

\section{ACKNOWLEDGMENTS}

The authors would like to thank all the reviewers of this paper for their valuable comments.

\section{REFERENCES}

[1] EIA/TIA. "Cellular radio-telecommunications intersystem operations," Tech. Rep. IS-41 Revision B, EIA/TIA, December 1991.

[2] M. Mouly and M. B. Pautet, "The GSM system for mobile communications," Telecom Publishing, Palaiseau, France, January 1992.

[3] J. Ho and I. Akyildiz, "Local anchor scheme for reducing signaling costs in personal communications networks," IEEE/ACM Transactions on Networks, vol. 4, no. 5, pp. 709725, October 1996.

[4] J. Ho and F. Akyildiz, "Local anchor scheme for reducing signaling costs in personal communications networks," IEEE/ACM Transactions on Networks, vol. 4, pp. 709-725, Oct. 1996.

[5] R. Jain and Y. B. Lin, "An auxiliary user location strategy employing forwarding pointer to reduce network impacts of PCS,"Wireless Networks., vol. 1, pp. 197-210, 1995.

[6] K. Kong, J. Gil, Y. Han, U. Song, and C. Hwang, "A forwarding pointer-based cache scheme for reducing location management cost in PCS networks," Journal of Information Science and Engineering, vol. 18, no. 5, pp. 1011-1025, 2002.

[7] W. Ma and Y. Fang, "Two-level pointer forwarding strategy for location management in PCS networks," IEEE Transactions on Mobile Computing, vol. 1, pp. 32-45, January - March 2002.

[8] W. Ma and Y. Fang "A pointer forwarding based local anchoring (POFLA) scheme for wireless networks," IEEE Transactions on Vehicular Technology, vol. 54, no. 3, May 2005.

[9] G. P. Pollini and C. I, "A profile-based location strategy and its performance," IEEE Journal on Selected Areas in Communications, vol. 15, no. 8, pp. 1415-1424, October, 1997.

[10] K. Kong, "Performance analysis of profile-based location caching with fixed local anchor for next-generation wireless networks," IEICE Transactions on Communications, vol. E91-B, no. 11, November 2008.

[11] Y. Fang, "Movement-based location management and tradeoff analysis for wireless mobile networks," IEEE Transactions on Computers, vol. 52, pp. 791-803, June 2003.

[12] Y. Fang, "General modeling and performance analysis for location management in wireless mobile networks," IEEE Transactions on Computers, vol. 51, pp. 1169-1181, October 2002.

[13] Y. Fang, I. Chlamtac, and Y. B. Lin, "Portable movement modeling for PCS networks," IEEE Transactions on Vehicular Technology, vol. 87, pp. 1347-1384, August 1999. 